\title{
Synergy between liposomal zoledronic acid and $\gamma \delta$ T-cells in the treatment of epithelial ovarian cancer
}

\author{
Ana Catarina Parente Pereira ${ }^{1 *}$, Hilary Shmeeda ${ }^{2}$, Lynsey Whilding ${ }^{3}$, Sadaf Ghaem-Maghami ${ }^{3}$, Alberto Gabizon ${ }^{2}$, \\ John Maher ${ }^{1}$
}

From Society for Immunotherapy of Cancer 28th Annual Meeting

National Harbor, MD, USA. 8-10 November 2013

$\gamma \delta$ T-cells contribute importantly to tumor immunosurveillance and are activated by phosphoantigen intermediates of the mevalonate pathway that are commonly over-produced in cancer cells. To potentiate this, phosphoantigen levels can be boosted using zoledronic acid (ZA). However, in vivo delivery of ZA to the tumour is inefficient owing to its poor pharmacokinetic properties. Here, we set out to develop a $\gamma \delta \mathrm{T}$-cell immunotherapy for epithelial ovarian cancer (EOC) using folate-targeted liposomal ZA (FT-L-ZA) to improve drug delivery to tumor cells. Folate receptor- $\alpha$ is over-expressed in EOC. Peripheral blood mononuclear cells isolated from healthy donors $(n=21)$ and EOC patients $(n=13)$ were cultured with ZA, IL-2 and IL-15 for two weeks. $\gamma \delta$ Tcells expanded reproducibly from healthy donors and patients, the latter having on average a 97 -fold expansion. The expanded $\gamma \delta \mathrm{T}$-cells expressed low levels of Lselectin, accompanied by CD45RO, CD27, CD70 and NKG2D, consistent with a combined central-memory and effector-memory phenotype, similarly to healthy controls. To evaluate whether ex-vivo cultured $\gamma \delta \mathrm{T}$ cells from patients with EOC are functionally competent, we developed an autologous co-cultivation assay whereby patient-derived $\gamma \delta$ T-cells were incubated invitro with primary EOC "tumorspheres". Twenty-four hours after incubation of tumorspheres with ZA, L-ZA or FT-L-ZA, ex-vivo expanded $\gamma \delta$ T-cells were added at a 1:100 ratio. When alone, $\gamma \delta \mathrm{T}$-cells destroyed $24.5 \%$ of spheres. Addition of $1 \mu \mathrm{g} / \mathrm{ml}$ free ZA sensitized tumorspheres to destruction by $\gamma \delta$ T-cells $(96.2 \%)$, but was relatively ineffective at $0.1 \mu \mathrm{g} / \mathrm{ml}(26.8 \%)$. By contrast,
FT-L-ZA was highly effective at $0.1 \mu \mathrm{g} / \mathrm{ml}(92.6 \%)$ while non-targeted L-ZA was no better than $\gamma \delta$ T-cells only. Destruction of ZA-sensitized tumorspheres by autologous $\gamma \delta \mathrm{T}$-cells was accompanied by their activation and production IFN $-\gamma(\approx 2000 \mathrm{pg} / \mathrm{mL})$. In the absence of $\gamma \delta$ $\mathrm{T}$-cells, none of these formulations exerted any toxic effect upon tumorspheres. To model EOC in vivo, SCID Beige mice were injected intraperitoneally (IP) with luciferase-expressing SKOV-3 cells. Mice ( $\mathrm{n}=5$ per group) were treated IP with $5 \mu \mathrm{g}$ ZA, FT-L-ZA or PBS, followed by $10 \mathrm{E} 7$ ex-vivo expanded $\gamma \delta \mathrm{T}$-cells from healthy controls or PBS. Tumour growth was monitored by bioluminescence. One week after treatment, mice treated with $\gamma \delta \mathrm{T}$-cells or $\gamma \delta \mathrm{T}$-cells and free ZA showed a tumour reduction of approximately $20 \%$. By contrast, mice treated with FT-L-ZA followed by $\gamma \delta$ T-cells achieved a tumour reduction of $60-70 \%$. Drugs alone had no therapeutic effect. We show here proof of concept for a synergistic immunotherapy for EOC whereby FT-L-ZA can be used to sensitize tumors to $\gamma \delta$ T-cells.

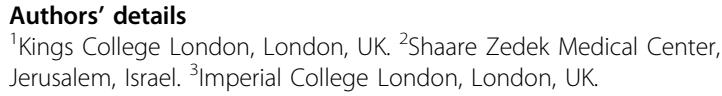

Published: 7 November 2013

doi:10.1186/2051-1426-1-S1-P28

Cite this article as: Pereira et al:: Synergy between liposomal zoledronic acid and $\gamma \delta$ T-cells in the treatment of epithelial ovarian cancer. Journal for ImmunoTherapy of Cancer 2013 1(Suppl 1):P28. 